\title{
Relationship Between Red Auroral Arcs and Ionospheric Recombination
}

\author{
G. A. M. King and F. E. Roach
}

(August 11, 1960)

\begin{abstract}
A "monochromatic" (6300 A) auroral arc, observed photometrically to be north of Boulder, Colo., has been identified with oblique echoes on ionograms taken near Boulder. The auroral emission is explained in terms of a great enhancement of ionospheric recombination. The recombination process is shown to depend on the density of molecular nitrogen in the ionospheric $F$ region; the change in nitrogen density during and after auroral activity, responsible for the change in recombination rate, is ascribed to the combined effects of heating and mixing of the atmosphere at the lower ionospheric heights.
\end{abstract}

\section{Introduction}

On the night of 27-28 November 1959, magnetic and auroral activity occurred over a large part of the United States. The general character of the activity can be judged from the variation of the horizontal component of the earth's magnetic field recorded at Boulder, Colo. (see fig. 1). Visual observers, especially in the northwestern United States, reported auroral structure during the evening, and, in particular, bright aurora with red in the northern sky was seen from Boulder about 0600 u.t. (Z).

Instrumental coverage of the period was excellent, and included (1) all-sky camera observations, (2) photometric observations of the green (5577 A) and the red $(6300 \mathrm{~A})$ auroral lines at three stations in the southwestern United States, (3) ionospheric soundings, including special recordings near Boulder in the low frequency range, $50 \mathrm{kc} / \mathrm{s}$ to $2 \mathrm{Mc} / \mathrm{s}$, and (4) measurement of fluxes of high-energy particles in the outer Van Allen zone during several passes of Explorer VII.

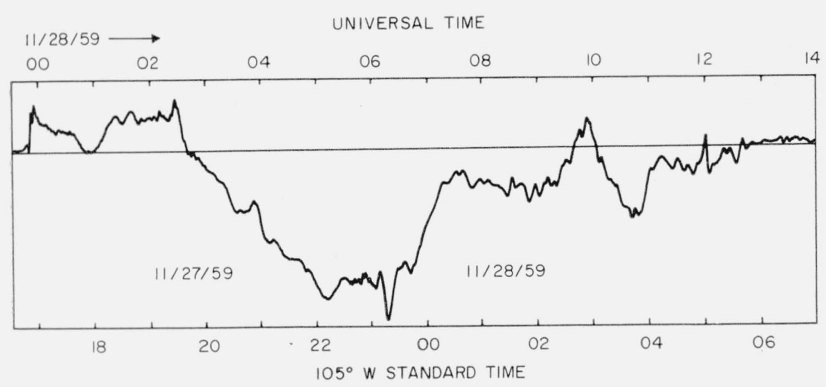

Figure 1. Plot of the horizontal component $(H)$ of the earth's magnetic field at Boulder during the 27-28 November 1959 period.

Full scale equals 330 gamma.

\footnotetext{
1 Contribution from Central Radio Propagation Laboratory, National Bureau
} of Standards, Boulder, Colo.
In this paper, we discuss the relationship between the photometric observations of a red arc and the ionospheric soundings. We hypothesize that the red emission is due to enhanced ionospheric recombination and draw semiquantitative support for the hypothesis from the observational data.

\section{Photometric Data}

The photometric equipment at Fritz Peak (near Boulder) was put into operation at $0410 \mathrm{Z}$ and continued in use throughout the night until $1310 \mathrm{Z}$. Observations were made with a birefringent filter photometer in the three colors, 5577 [OI], $6300[\mathrm{OI}]$, and $5893[\mathrm{NaI}]$. The forbidden oxygen radiations (5577 and 6300) were active throughout the night. Two classes of activity occurred, and for convenience we will classify them as follows:

(1) $R G$. In this class, both the red (6300) and the green (5577) emissions are enhanced in intensity.

(2) $R$. In this class the emission forms an arc which is selectively enhanced in the red with no apparent increase in the intensity of the green emission.

It is tempting to think of $R G$ as regular auroral activity, since both the red and green lines are characteristic features of auroras in general. The properties of $R$-arcs are described in several recent papers; Barbier $[1]^{2}$ discussed their occurrence and general nature, while Roach and Marovich [2, 3] gave detailed descriptions of two $R$-arcs.

On this particular night, the $R G$ activity covered a wide range of intensity (see fig. 2) whereas the $R$ activity went through slow and ponderous changes. The general impression from figure 2 is that after $0700 \mathrm{Z}$, when the $R$ data were no longer contaminated by the strong $R G$ background, there was a tendency of monotonic decay in the $R$ activity with a resurgence at $0925 \mathrm{Z}$.

${ }^{2}$ Italicized figures in brackets indicate the literature references at the end of this paper. 


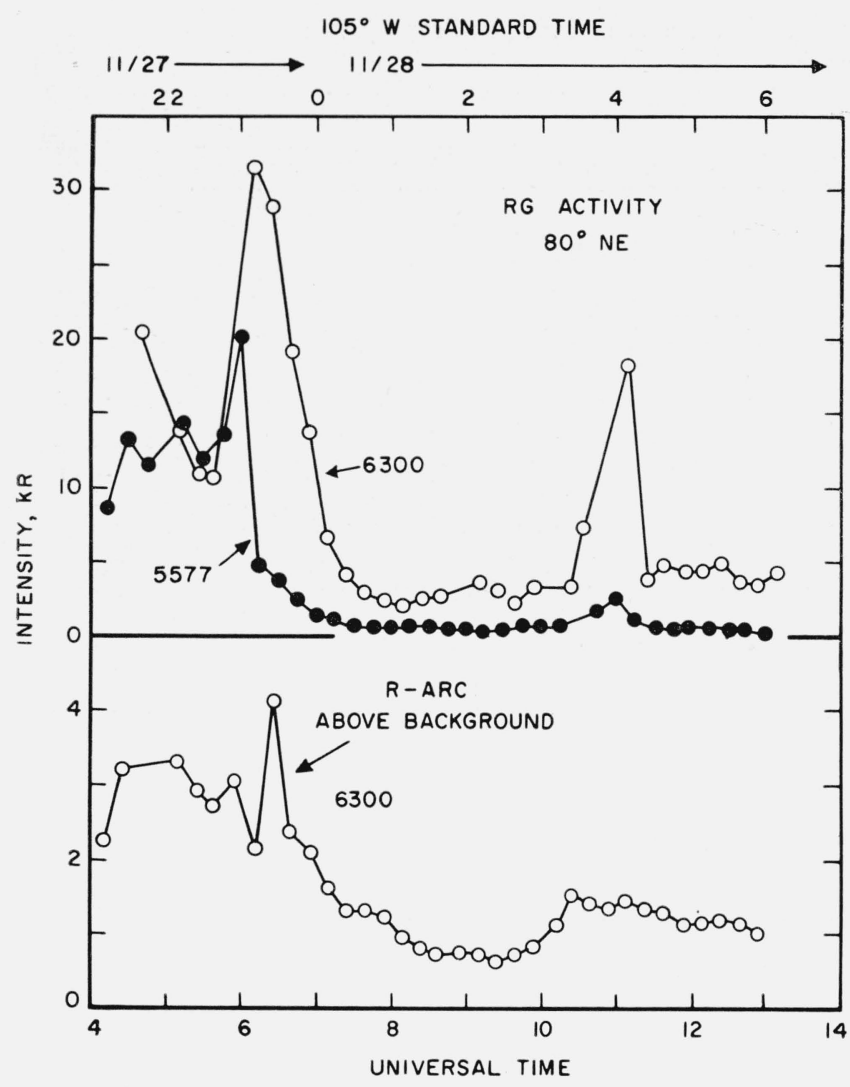

FIgure 2. Intensity changes of the RG activity near the northeast horizon (above) and of the $R$-arc (below) during the night of 27-28 November 1959.

The height and geographical position of the $R$-arc have been determined in a cooperative study by workers from Fritz Peak (Colo.), Cactus Peak (Calif.), and Sacramento Peak (N. Mex.) [4]. By triangulation, they find a mean height of about $400 \mathrm{~km}$. With this value and the angular data from the Fritz Peak records the arc's geographical position has been deduced; the results are shown in figure 3. At the same time, its width in latitude was determined to be about $500-600 \mathrm{~km}$.

\section{Ionospheric Data}

Ionospheric records were taken half-hourly at Boulder, $26 \mathrm{~km}$ from Fritz Peak. Figure 4 shows the record at $0730 \mathrm{Z}$, together with a diagram setting out the salient features of the ordinary wave reflections.

A conspicuous feature of this ionogram is the spread echo above the main trace. As the $F$ region totally reflects all waves traveling vertically in this frequency range, it is not possible that the spread echo be overhead at a greater height than the normal layer; therefore, the spread trace must be due to echoes received obliquely from a large irregularity in the $F$ layer some distance from the observing station. Further considerations, based on the conditions necessary for reflection, suggest that the irregularity is at about the same height as the layer giving the vertical echoes.

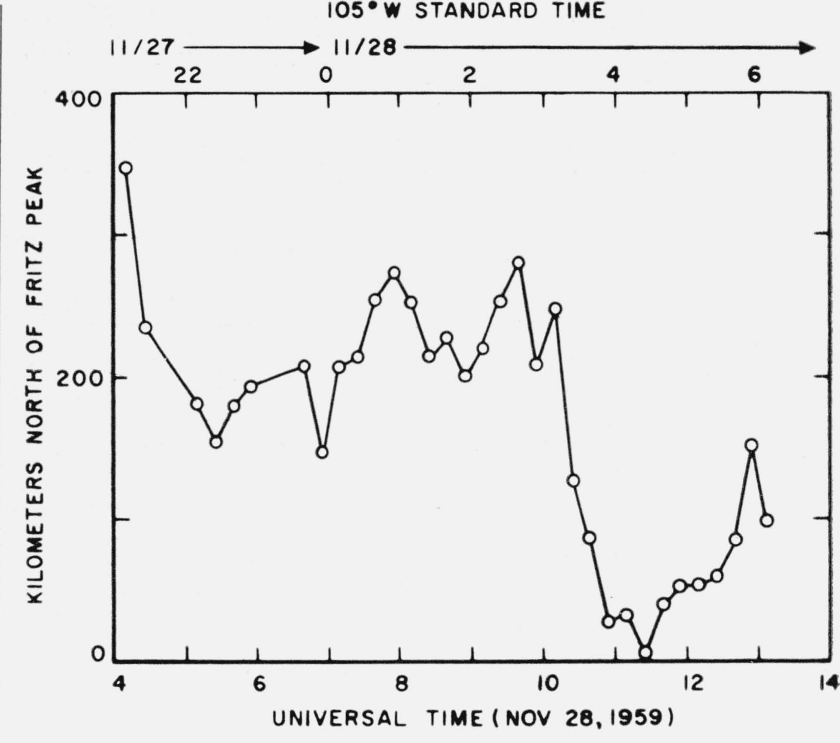

Figure 3. Position of the R-arc of 27-28 November 1959 with respect to Fritz Peak

Ordinate indicates kilometers north of Fritz Peak for an assumed height of $400 \mathrm{~km}$

Unfortunately, because of the large effects of ionospheric refraction, it is not possible to determine the distance to the irregularity. However, these refraction effects (which also determine the virtual height, $h^{\prime}$, of the vertical reflection) will to some extent be canceled when we take the ratio of the virtual ranges to the oblique and to the overhead echoes. A very crude measure, then, of the zenith angle, $z$, of the irregularity is given by,

$$
\sec z=1+\frac{\Delta s}{h^{\prime}}
$$

where $\Delta s$ is indicated on figure 4 and $h^{\prime}$ is the virtual height of the overhead echo at $2 \mathrm{Mc} / \mathrm{s}$ where the results are least affected by differences between the electron distribution in the irregularity and in the overhead $F$ layer. This "zenith angle" was determined from all the ionograms between 0700 and $1200 \mathrm{Z}$, and it is plotted on figure 5 together with the angle to the $R$-arc determined directly from the photometric records. The parallelism of the two curves suggests immediately that the irregularity and the $R$-arc are associated, especially in view of the fact that the height of the arc places it in the $F$ region. This conclusion has been reinforced by a study of the temporal association of oblique echoes and $R$-arcs, using ionograms from Fort Monmouth, White Sands, and Boulder, for nights in September 1957 and December 1959.

An important observation which can be made from figure 4 is that the electron density in the irregularity is less than in the overhead trace. In other words, the $R$-arc is associated with a local decrease in the ionization of the $F$ region. 

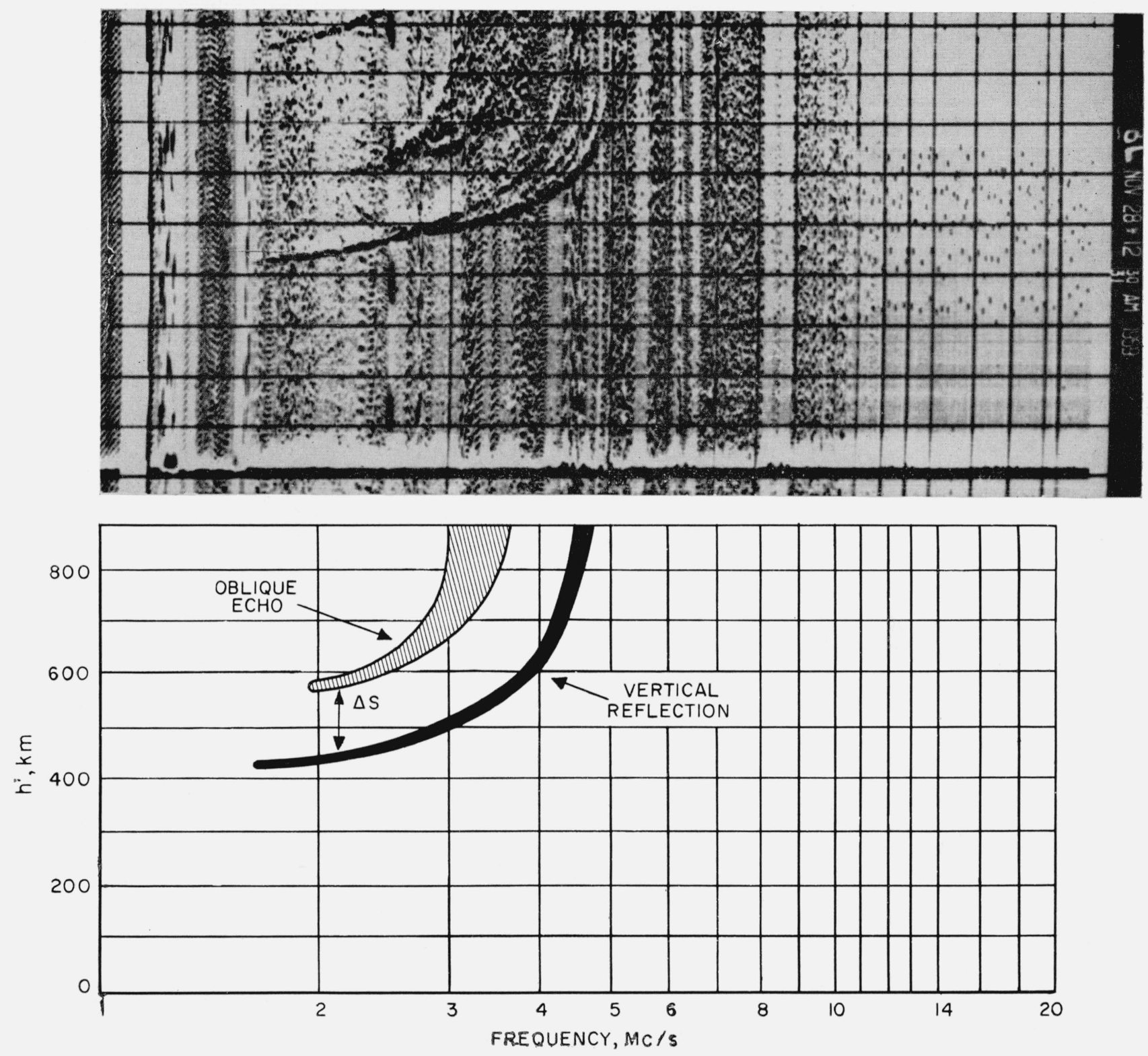

Figure 4. Ionogram made at Boulder at $0730 \mathrm{Z}$.

The lower diagram illustrates the essential features.

For testing the theoretical deductions discussed later, we needed electron density profiles. Fortunately, besides the normal ionograms covering frequencies above $1 \mathrm{Mc} / \mathrm{s}$, there were special records over the frequency range $50 \mathrm{kc} / \mathrm{s}$ to $2 \mathrm{Mc} / \mathrm{s}$, made at a field station $23 \mathrm{~km}$ from Boulder and $13 \mathrm{~km}$ from Fritz Peak. By using both sets of records, we were able to get profiles by real height analysis free from the serious errors of "unobserved ionization." The most reliable profile was that at $0730 \mathrm{Z}$, as the traces on the ionogram were well defined (see fig. 4). From 0800 to $1000 \mathrm{Z}$ profiles could not be obtained, because of sporadic $E$, and after $1030 \mathrm{Z}$ the traces were diffuse, so that the upper parts of the profiles were unreliable. It must be noted that the electron density profiles apply to the main trace and not to conditions in the region of the irregularity, for which they cannot be obtained.

\section{Red Emission Due to Ionospheric Recombination}

We will attempt to explain four observational facts. which have been presented above:

(1) Occurrence of the $R$-arc after the main phase of the magnetic disturbance.

(2) Steady change of intensity with time.

(3) Strong emission of the red lines of atomic oxygen without measurable emission of the green oxygen line. 


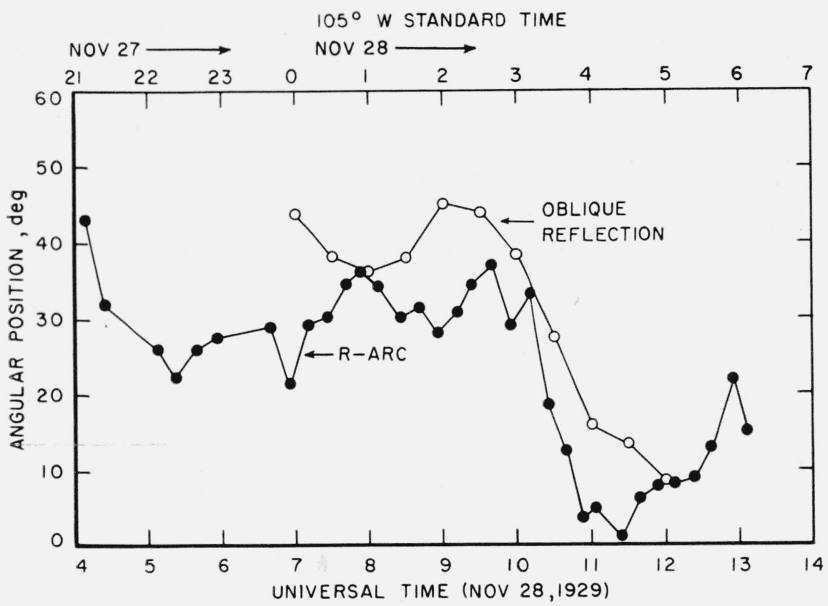

Figure 5. Comparison of the angular position of the R-arc and the oblique echo during part of the night of 27-28 $\mathrm{No}$ vember 1959 .

(4) Association with a local decrease in the ionization of the $F$ region.

Consider the normal process of recombination in the ionosphere. The ionospheric electrons are produced during the daylight hours by photoionization of atomic oxygen.

$$
\mathrm{O}+h \nu \rightarrow 0^{+}+e .
$$

As the reverse process of radiative recombination is extremely slow, the removal of electrons, both by day and by night, occurs through a two step process [5]. This involves first atom-ion interchange between the ionized oxygen atom and molecular nitrogen,

$$
\mathrm{O}^{+}+\mathrm{N}_{2} \rightarrow \mathrm{NO}^{+}+\mathrm{N}
$$

and then dissociative recombination,

$$
\mathrm{NO}^{+}+e \rightarrow \mathrm{N}^{*}+\mathrm{O}
$$

or

$$
\mathrm{NO}^{+}+e \rightarrow \mathrm{N}+\mathrm{O}^{*} \text {. }
$$

(Similar reactions involving molecular oxygen will be discussed later and shown to be unimportant for the present purpose.)

Equation (4) gives a relationship between recombination and upper atmospheric emissions; the products of the recombination are excited atoms of nitrogen or oxygen, and when these fall to the ground states they emit characteristic radiations at $5199[\mathrm{NI}]$ and 6300 (and 6364) [OI].

Given the distributions with height of the electrons and of molecular nitrogen, one can compute the rate of electron recombination, assuming that $\mathrm{NO}^{+}$is in equilibrium between reactions (3) and (4). The approximate equation is $[6]$.

$$
\frac{\partial n}{\partial t}=-\frac{\alpha n \gamma\left[N_{2}\right]}{\alpha n+\gamma\left[N_{2}\right]} \cdot n
$$

where

$n$ is the electron density

$\left[\mathrm{N}_{2}\right]$ is the number density of $\mathrm{N}_{2}$ $\alpha$ is the rate coefficient of reaction (4) $\gamma$ is the rate coefficient of reaction (3).

The exothermic energy balance of eq (4) is $2.7 \mathrm{ev}$, sufficient to provide either an NI quantum (2.36 ev) or an OI quantum (1.96 ev) but not both. Lacking precise data on the relative probability of (4a) and (4b), we shall assume that one OI quantum is emitted for every two recombinations, i.e.,

$$
E \approx-\frac{1}{2} \frac{\partial n}{\partial t}
$$

As an illustration, we will apply the above theory to the calculation of the red emission on a quiet night [7]. The time chosen is $0730 \mathrm{Z}, 19$ November 1958 , and the electron density profile is deduced from the Boulder ionograms. The $\left[N_{2}\right]$ profile is derived from atmospheric densities given by Kallmann and Juncosa [8], assuming that the gases are in diffusive equilibrium, and normalized so that $\gamma\left[\mathrm{N}_{2}\right]$ at $300 \mathrm{~km}$ equals the recombination rate determined by Ratcliffe et al. [9],

$$
\beta_{300}=\gamma\left[N_{2}\right]_{300}=10^{-4} / \mathrm{sec} .
$$

For $\alpha$, we adopt

$$
\alpha=2 \times 10^{-8} \mathrm{~cm}^{3} / \mathrm{sec} .
$$

The electron density profile and the corresponding OI emissions are shown together in figure 6 . The integrated emission of 28 rayleighs agrees well with observed values on quiet nights, and in fact Barbier [10] gives a value of 30 rayleighs for $0700 \mathrm{Z}$ at Haute Provence on this night (Fritz Peak data are not available for the time in question). The height of the nightglow, 6300, has not vet been measured, but the evidence places it in the $F$ region $[11,12]$. The height of maximum emission, $260 \mathrm{~km}$, deduced in this example is very reasonable.

We will now see if the same theory can explain the $R$-arc on the night of 28 November 1959 .

Two additional factors must be considered when converting the calculated values of $\partial n / \partial t$ into emission of 6300 quanta. One is that electron densities in the region of the arc are less than in the overhead $F$ region, vet the latter are used in the computation. The other factor arises from the shape of the arc, broad in comparison with its vertical extent; as the observations were not made vertically, the measured values are larger than those for vertical observation. Neither factor is large, and they are in opposite senses; so, for convenience, we shall assume that they cancel and that a direct comparison can be made between the theory based on eqs (5) and (6) and uncorrected observations.

We suppose also that the abnormally great brilliance of the $R$-arc is due to enhanced recombination: i.e., that $\beta$ is larger than its normal value in such a ratio that the calculated emission agrees with the observed emission. The test of the theory, then, is based on comparison of the predicted and the observed heights of maximum emission. 


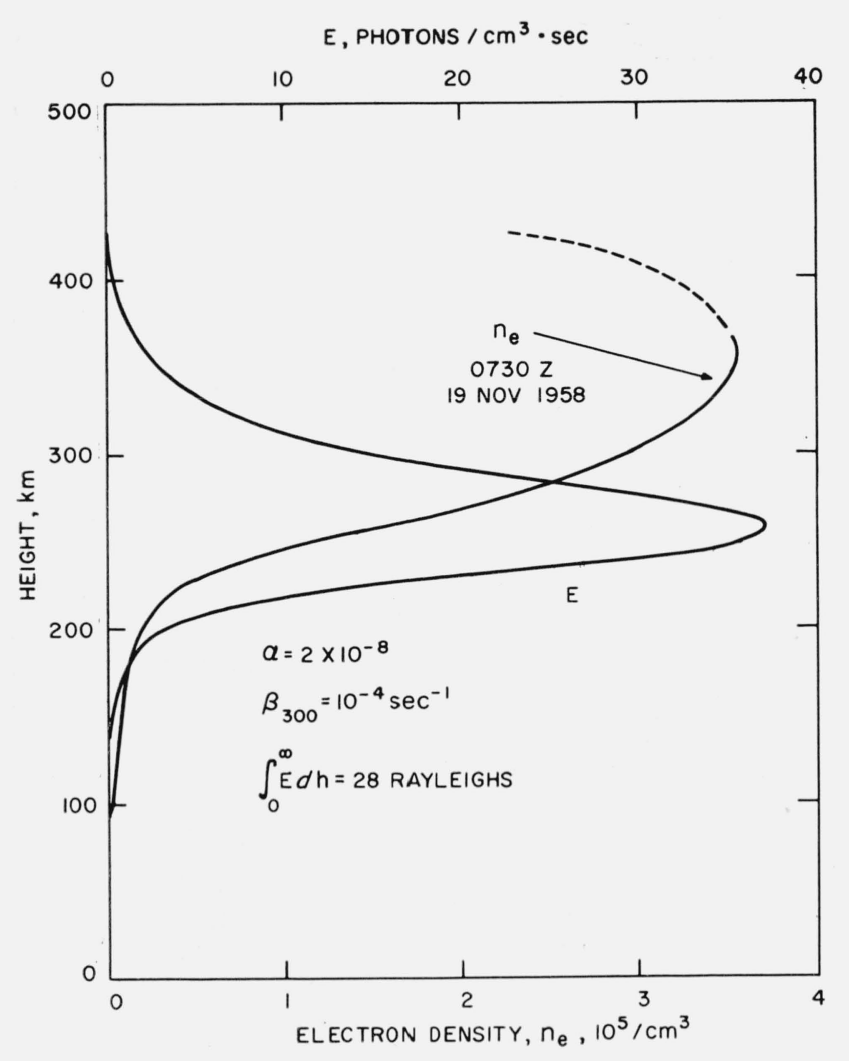

Figure 6. Electron density profile $\left(\mathrm{n}_{\mathrm{e}}\right)$ and the 6300 emission profile (E) under quiet conditions.

For the electron density profile at $0730 \mathrm{Z}$ almost perfect agreement is reached if $\beta_{300}=10^{-1} / \mathrm{sec}$. The electron profile and the emission profile are shown together in figure 7 . Figure 8 presents the comparisons for the five times studied; in each comparison the curved line gives the theoretical relation between integrated emission and height of maximum emission as $\beta$ goes through a range of values, while the circles connected by a heavy line give the observed points, corrected and not corrected respectively for background $R G$ activity. As in the $0730 \mathrm{Z}$ case, the nearest approach to agreement occurs when $\beta_{300}$ is about $10^{-1} / \mathrm{sec}$.

The effect of varying $\alpha$ between $10^{-8}$ and $10^{-7} \mathrm{~cm}^{3}$ sec was studied, and $2 \times 10^{-8}$ gave the best overall agreement.

\section{Reason for the Increased Recombination}

The rate of recombination required to give agreement between the theory and the observations is a thousand times the normal rate. This factor is so large that, in spite of the fair sucess of the theory, we must ask if it is reasonable.

The recombination rate, $\beta$, is proportional to $\left[\mathrm{N}_{2}\right]$ (see eq (7)). Normally, at $300 \mathrm{~km},\left[\mathrm{~N}_{2}\right]$ is about a thirtieth of the total number of atmospheric particles; this figure is based on a diffusive level of $105 \mathrm{~km}$ in agreement with rocket observations [13].

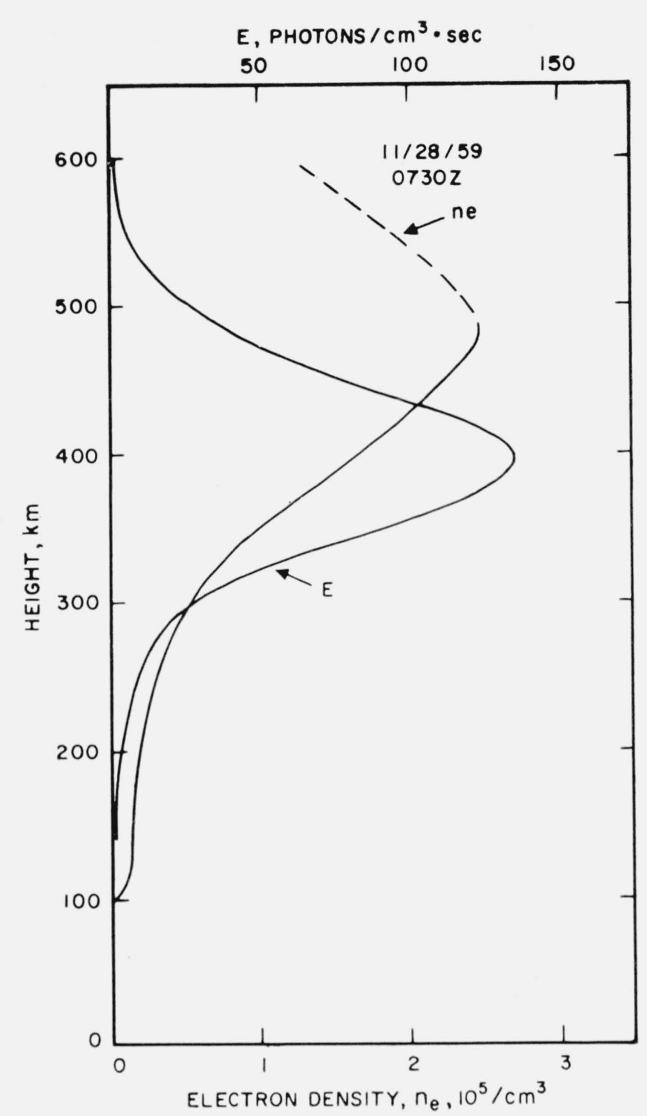

FIgURE 7. Electron density profile $\left(\mathrm{n}_{\mathrm{e}}\right)$ and the 6300 emission profile (E) at $0730 \mathrm{Z}$ on the night of 28 November 1959. $\alpha=2 \times 10^{-8} / \mathrm{cm}^{3} \mathrm{sec}$ and $\beta_{300}=10^{-1} / \mathrm{sec}$.

The integrated emission is 1460 rayleighs.

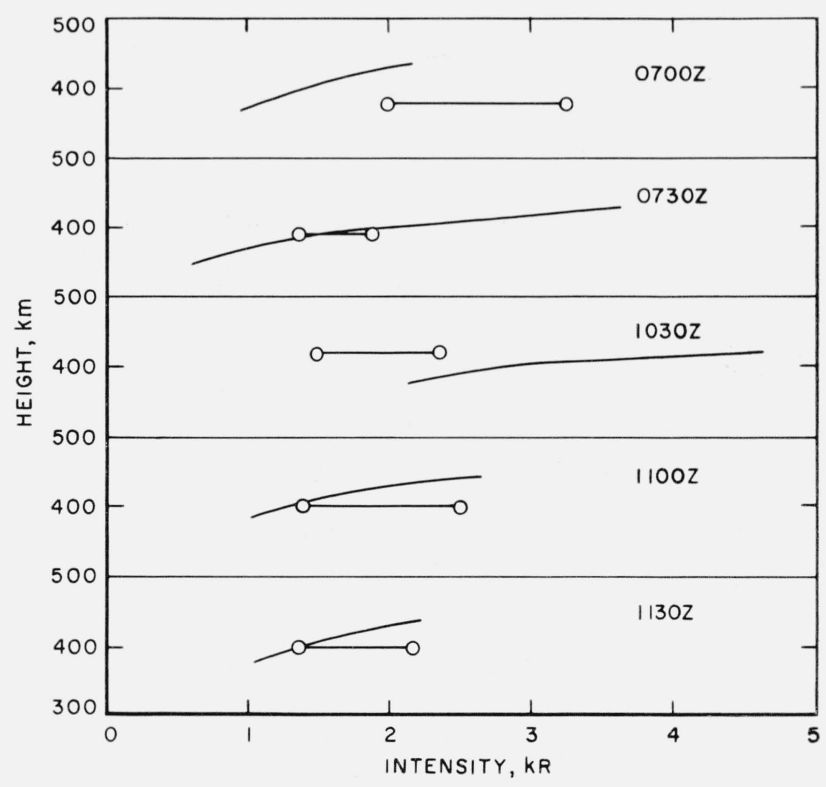

Figure 8. Comparison between theory and observation at five times during the night of 28 November 1959.

(See text) 
However, during auroral activity the atmosphere is heated by the influx of energetic particles. Direct evidence of this has recently been provided by Mulyarchik [14] who measured the temperature of the region emitting the 6300 OI line; he found that the temperature increased with the brightness of the display. Now, under normal conditions, the $F$ region is hotter than $E$. But most of the energy of the aurora is set free in the $E$ region, and, if the increase in $F$-region temperature is due to conduction of heat from the $E$ region, the normal temperature gradient would have to be reversed. This would call for a very great rise in the $E$-region temperature, and consequent great changes in atmosphere densities at all higher levels. Another consequence of interest here is the mixing caused by the reversal of the temperature gradient; this raises the diffusive level and so increases the fractional molecular concentration at higher levels. Thus, during and after auroral activity, we expect to find a great increase in $\left[\mathrm{N}_{2}\right]$ at $F$-region heights, and a factor of a thousand does not seem unreasonable.

Three other facts deserve mention in this respect. The first is that the electron density profiles on 28 November 1959 are consistent with atmospheric scale heights in the $F$ region one and a half times normal (compare figs. 6 and 7). Next, satellite drag data indicate an increase in atmospheric densities at times of magnetic storms [15]. Finally, Lytle and Hunten [16] report that at dawn after an active aurora the $\mathrm{N}_{2}^{+}$emission was very greatly enhanced; while they explain this simply in terms of resonance scattering from $\mathrm{N}_{2}^{+}$ions generated by the aurora, it seems to us more likely in view of the rapid recombination of $\mathrm{N}_{2}^{+}$that the ions were generated by solar ultraviolet light, and that the difference between this and normal dawn lies in the increased $\left[\mathrm{N}_{2}\right]$.

\section{Further Discussion}

Accepting the ideas of the last section, we must ask what effect such a large change in the atmosphere would have on the computation of 6300 emission according to the theory outlined previously; clearly, the $\left[\mathrm{N}_{2}\right]$ profile should not be derived in the simple manner used for quiet night conditions. A trial calculation, based on scale heights greater by a factor of one and a half than those of Kallmann and Juncosa [8], shows that the emission profile is thickened, but that the height of maximum emission is not greatly altered; more important, however, is that the value of $\beta_{300}$ needed to give the observed emission is decreased to only 400 times the normal value. As mixing also increases the effective scale height of $\left[\mathrm{N}_{2}\right]$, it is likely that on the night of $27-28$ November 1959, the increase in $\beta_{300}$ was much less than the thousand times deduced earlier. The data do not justify a more complete analysis to determine the increase precisely.

When discussing the theory of recombination, we based it on reactions with molecular nitrogen rather than with molecular oxygen as has been supposed by other workers [7]. The relevant equations are

$$
\begin{aligned}
& \mathrm{O}^{+}+\mathrm{O}_{2} \rightarrow \mathrm{O}_{2}^{+}+\mathrm{O} \\
& \mathrm{O}_{2}^{+}+e \rightarrow \mathrm{O}^{*}+\mathrm{O}^{* *} .
\end{aligned}
$$

We have three reasons for believing that reactions (9) and (10) are not those of interest in the present case. The first is that Dickinson and Sayers [17] have recently measured the rate coefficient of reaction (9), obtaining the value $2.5 \times 10^{-11} \mathrm{~cm}^{3} / \mathrm{sec}$; if diffusive separation of the atmospheric gases starts at $105 \mathrm{~km}[13]$, then $\left[\mathrm{O}_{2}\right]_{300}$ is only about $5 \times 10^{5} / \mathrm{cm}^{3}$, which with the measured rate coefficient is much too small to give the observed ionospheric recombination rate. Secondly, the exothermic energy balance of eq (10) is $6.9 \mathrm{ev}$, enough to give the oxygen green line $(4.2 \mathrm{ev})$ as well as the red lines; whereas there is virtually no green emission in the $R$-arcs [3]. Finally, the temperature measurements mentioned earlier [14] also included observations of the NI lines at 5199, and the values are in the same range as those for the OI 6300 , indicating that both emissions are from the same height; the reactions involving molecular nitrogen yield both NI 5199 and OI 6300 (see eq (4)). It seems that the $R$-ares are not truly "monochromatic," but are only considered so because photometric observations of 5199 are not available.

It is worth mentioning that the rate coefficient of the atom-ion interchange reaction with molecular nitrogen (eq (3)) has been reported in the literature [18], but the value, $10^{-8} \mathrm{~cm}^{3} / \mathrm{sec}$, is almost certainly incorrect. The laboratory experiment was for the purpose of testing a mass spectometer, and the rate coefficient was an incidental result; it is likely that no precautions were taken to exclude contamination from the apparatus.

\section{Summary}

A hypothesis is submitted for the excitation in the upper atmosphere of the oxygen atom to the ${ }^{1} \mathrm{D}$ state. The proposed mechanism involves (1) an atom-ion interchange in which $\mathrm{O}^{+}$and $\mathrm{N}_{2}$ go to $\mathrm{NO}^{+}$and $\mathrm{N}$ and (2) a dissociative recombination in which $\mathrm{NO}^{+}$and an electron yield excited atomic nitrogen or oxygen. The dissociative recombination is sufficiently exothermic $(2.72 \mathrm{ev})$ to excite the oxygen atom to the ${ }^{1} \mathrm{D}$ state $(1.96 \mathrm{ev})$ but not to the ${ }^{1} \mathrm{~S}$ state $(4.16 \mathrm{ev})$.

To account for the strong emission of [OI] 6300 in the form of arcs on the equatorward side of general auroral activity it is hypothesized that during an aurora, the rate of the atom-ion interchange is increased by a large increase in the density of molecular nitrogen at $F$-region heights as a consequence of heating and mixing of the atmosphere below. The predictions of the hypothesis are in agreement with the observed height and intensity of the $R$-arc on the night of $27-28$ November 1959 . 
One of us (G. A. M. K.) wishes to acknowledge support under the International Geophysical Year program of the National Academy of Sciences while he was a guest worker at the High Altitude Observatory and at the National Bureau of Standards, Boulder, Colo.

\section{References}

[1] D. Barbier, L'activite aurorale aux bases latitudes, Ann Geophys. 14, 334 (1958).

[2] F. E. Roach and E. Marovich, A monochromatic low latitude aurora, J. Research NBS 63D, 297 (1959).

[3] F. E. Roach and E. Marovich, Aurora of October 22/23, 1958, at Rapid City, South Dakota, J. Research NBS 64D, 205 (1960).

[4] F. E. Roach, J. G. Moore, E. C. Bruner, H. Cronin, and S. M. Silverman, The height of maximum luminosity in an auroral arc, J. Geophys. Research 65, $3575^{\circ}(1960)$.

[5] D. R. Bates and H. S. W. Massey, The basic reactions in the upper atmosphere, Proc. Roy. Soc. (London) 192, 1 (1947).

[6] J. A. Ratcliffe, Formation of the ionospheric layers $\mathrm{F}_{1}$ and $\mathrm{F}_{2}$, J. Atmospheric and Terrest. Phys. 8, 260 (1956).

[7] J. W. Chamberlain, Oxygen red lines in the airglow: I. Twilight and night excitation processes, Ap. J. 127, $54(1958)$

[8] H. K. Kallmann and M. L. Juncosa, A preliminary model atmosphere based on rocket and satellite data, Rand Rept. RM 2286 (1958).
[9] J. A. Ratcliffe, E. R. Schmerling, C. S. Setty, and J. O. Thomas, The rates of production and loss of electrons in the F-region of the ionosphere, Phil. Trans. Roy. Soc. A248, 609 (1956).

[10] D. Barbier, report to the IGY data centers.

[11] P. St. Amand, Some possible relations between the nightglow and the ionosphere, Ann. de Geophysique 11, 450 (1955).

[12] M. J. Koomen, R. Scolnik, and R. Tousey, Direct measurement of the night airglow, "The Threshold of Space" 211 (Pergamon 1957).

[13] E. B. Meadows and J. W. Townsend, Diffusive Separation in the Winter Nighttime Arctic Upper Atmosphere, Ann. de Geophysique 14, 80 (1958).

[14] T. M. Mulyarchik, Interferometric temperature measurements of the upper atmosphere, based on the width of certain emission lines, Izvestiya Akademii Nauk SSSR, Ser. Geofiz. 3, 449 (1960).

[15] L. G. Jacchia, Corpuscular radiation and the acceleration of artificial satellites, Nature 183, 1662 (1959).

[16] E. A. Lytle and D. M. Hunten, Dawn enhancement of auroral $\mathrm{N}_{2}{ }^{+}$emission, Can. J. Phys. 38, 477 (1960).

[17] P. H. G. Dickinson and J. Sayers, Ion charge exchange reactions in oxygen afterglows, Proc. Phys. Soc. (in press).

[18] R. F. Potter, Atom-ion interchange in air, J. Chem. Phys. 23, 2462 (1955).

(Paper 65D2-110) 